\title{
Diabetes mellitus tipo 1 y condición periodontal
}

\author{
Jonathan Castaño, Est. ${ }_{1}$, Alexander Ortega, Est. ${ }_{1}$, Jonathan Ortega, Est. ${ }_{1}$, Julián Mauricio Palacios, Est. \\ Adolfo Contreras*, Ph.D.
}

${ }_{1}$ Escuela de Odontología, Universidad del Valle, Cali, Colombia

Recibido: 15 de diciembre del 2013. Aprobado: 14 de mayo del 2014.

*Autor de correspondencia: Adolfo Contreras, Escuela de Odontología, Universidad del Valle, Cali, Colombia. Calle 4 B n. ${ }^{0} 36$ - 00, teléfono (57) 25579005 , correo electrónico: adolfoco@yahoo.com

Cómo citar este artículo: Castaño J, Ortega A, Ortega J, Palacios JM, Contreras A. Diabetes mellitus tipo 1 y condición periodontal. Rev Nac Odontol. 2014; 10(18):7-14. doi: http://dx.doi.org/10.16925/od.v10i18.716

Resumen. Introducción: la diabetes tipo 1 (DM1) afecta el metabolismo de glucosa, lípidos y proteínas e incrementa el riesgo de enfermedad periodontal. El objetivo de esta investigación fue identificar las características periodontales en pacientes con diagnóstico de DM1. Materiales y métodos: se estudiaron 35 pacientes con diabetes tipo 1, a quienes se les realizó un examen periodontal completo; se tomaron los valores de hemoglobina glicosilada (HbA1C) y de glicemia en ayunas; los datos se analizaron de forma descriptiva y comparativa contra los parámetros clínicos periodontales y el diagnóstico periodontal. Resultados: la prevalencia de periodontitis crónica fue de $74,3 \%$ y de gingivitis, de $25,7 \%$. El valor promedio de hemoglobina glicosilada (HbA1C) de los pacientes diagnosticados con gingivitis fue de 9,15, mientras que en pacientes con periodontitis fue de 8,375, sin alcanzar significancia estadística. La pérdida de inserción clínica periodontal en promedio fue de $3,15 \mathrm{~mm}$, mientras que la media del número de dientes perdidos fue 6,91 para toda la población. Los pacientes con periodontitis presentaron mayor pérdida de inserción clínica y profundidad de bolsa. Conclusiones: los pacientes con DM1 tuvieron periodontitis o gingivitis, ratificando su susceptibilidad de sufrir periodontitis y deben mantener un cuidadoso control gingival y periodontal.

Palabras clave: diabetes mellitus tipo 1, gingivitis, glicemia, hemoglobina glicosilada, periodontitis.

\section{Type 1 diabetes mellitus and periodontal disease}

Abstract. Introduction: Type 1 diabetes (DM1) affects the metabolism of glucose, lipids and proteins and increases the risk of periodontal disease. The aim of this research was to identify the periodontal characteristics of patients diagnosed with DM1. Materials and methods: the study examined 35 patients with DM1, who were given a complete periodontal exam; fasting values of glycated hemoglobin (HbA1C) and glycemia were taken and the data were analyzed both descriptively and comparatively against the periodontal clinical parameters and the periodontal diagnosis. Results: the prevalence of chronic periodontitis was $74.3 \%$ and of gingivitis, $25.7 \%$. The average value of glycated hemoglobin $(\mathrm{HbAlC})$ for patients diagnosed with gingivitis was 9.15 , while for patients with periodontitis it was 8.375 , without being statistically significant. Average periodontal clinical attachment loss was $3.15 \mathrm{~mm}$, while the average number of teeth lost was 6.91 for the entire population. The patients with periodontitis presented greater clinical attachment loss and pocket depth. Conclusions: the patients with DM1 had periodontitis or gingivitis, confirming their susceptibility to periodontitis, and they should therefore take care to receive regular gingival and periodontal check-ups.

Keywords: Type 1 diabetes mellitus, gingivitis, glycemia, glycated hemoglobin, periodontitis.

\section{Diabetes mellitus tipo 1 e condição periodontal}

Resumo. Introdução: A diabetes tipo 1 (DM1) afeta o metabolismo da glicose, lipídios e proteínas e aumenta o risco de doenças periodontal. $\mathrm{O}$ objetivo desta pesquisa foi identificar as características periodontais em pacientes com diagnóstico de DM1. Materiais e métodos: estudaram-se 35 pacientes com diabetes tipo 1 , aos que realizaram um exame periodontal completo; tiraram os valores de hemoglobina glicosilada (HbAlC) e de glicemia em jejum; os dados foram analisados de forma descritiva e comparativa contra os parâmetros clínicos periodontais e o diagnóstico periodontal. Resultados: a prevalência de periodontite crônica foi de $74,3 \%$ e de gengivite de $25,7 \%$. O valor médio de hemoglobina glicosilada (HbA1C) dos pacientes diagnosticados com gengivite foi de 9,15, enquanto em pacientes com periodontite foi de 8,375, sem alcançar significância estatística. A perda de inserção clínica periodontal em média foi de $3,15 \mathrm{~mm}$, enquanto a média do número de dentes perdidos foi 6,91 para toda a população. Os pacientes com periodontite apresentaram maior perda de inserção clínica e profundidade de bolsa. Conclusões: os pacientes com DM1 tiveram periodontite ou gengivite, ratificando sua suscetibilidade de sofrer periodontite e devem manter um cuidadoso controle gengival e periodontal.

Palavras-chave: diabetes mellitus tipo 1, gengivite, glicemia, hemoglobina glicosilada, periodontite. 


\section{Introducción}

La diabetes es una enfermedad sistémica que afecta principalmente el metabolismo de la glucosa, pero también el de lípidos y proteínas del organismo y se divide en diabetes mellitus tipo 1 o DM1, que es más severa porque genera más dependencia de la insulina y aparece en personas más jóvenes, y la diabetes mellitus tipo 2 o DM2, que se asocia con un padecimiento más crónico, aparece más tarde en el ciclo de vida y se asocia con sobrepeso y obesidad, entre otros factores de riesgo [1-3]. Se estima que en Colombia la prevalencia de diabetes en general puede ser de $8 \%$ aunque aparecen menos casos de DM1 [4]. La diabetes mellitus tipo 1 es consecuencia de interacciones de factores genéticos, ambientales e inmunológicos, que culmina en la destrucción de células beta del páncreas y la deficiencia de insulina. Algunos autores, no obstante, explican que los individuos que la padecen expresan deficiencia de la insulina por mecanismos no inmunitarios desconocidos y fácilmente presentan ceto-acidosis.

En cuanto a la enfermedad periodontal, es una patología de carácter crónico progresivo en la que se altera el tejido de soporte que rodea a la estructura dentaria e incluye el cemento radicular, el hueso alveolar y los tejidos blandos como el tejido conectivo gingival y periodontal. Su etiopatogenia es la presencia de bacterias Porphyromonas gingivalis, Tannerella forsythia, Treponema denticola, Prevotella intermedia, P. nigrescens y Aggregatibacter actinomycetemcomitans, entre otras, en un huésped susceptible por la acumulación de placa bacteriana dental o biopelícula dental a causa de la deficiente higiene oral. Es de vital importancia el estudio de estas dos patologías porque al parecer tienen una relación de dos vías [5]. En otras palabras, la DM1 incrementa en los sujetos la susceptibilidad de desarrollar periodontitis $y$, por otro lado, la enfermedad periodontal no tratada afecta el metabolismo de la glucosa agravando la condición de diabetes. Por último, se conoce que el tratamiento periodontal mejora el control de la glucosa en los pacientes con diabetes, posiblemente por una reducción de la infección y la inflamación. En diversos estudios se han encontrado marcadores clínicos periodontales incrementados tales como profundidad de bolsa, sangrado al sondaje y pérdida de inserción periodontal en pacientes con DM1 [6-8]. Con lo anterior, es fundamental que el profesional de odontología conozca la patogenia de estas entidades y la relación que presentan, para realizar un buen manejo integral y para establecer las interrelaciones de forma eficaz con los médicos tratantes y con el grupo de soporte familiar por el carácter crónico y progresivo de la diabetes. En Colombia, el incremento del sedentarismo y la obesidad en los jóvenes ha aumentado el riesgo de padecer diabetes. Esto amerita concientización de la población en general y de los jóvenes en particular; además, urge la prevención y el tratamiento de la diabetes por parte de los profesionales de la salud.

\section{Métodos}

La diabetes mellitus es un desorden metabólico que afecta la asimilación de los hidratos de carbono y de las proteínas, principalmente. Se dividen básicamente en: diabetes mellitus tipo 1, cuya principal característica es la producción de anticuerpos contra las células beta del páncreas, y a la que también se conoce como diabetes juvenil, pues aparece en niños y jóvenes y parece que tiene un riesgo genético o se dispara como consecuencia de una infección severa; y diabetes mellitus tipo 2, que es asociada a condiciones como obesidad y sobrepeso y aparece con mayor frecuencia en los adultos. Otros tipos de diabetes menos frecuentes son: la diabetes idiopática, que es similar a una diabetes tipo 1, pero sin anticuerpos contra las células beta del páncreas y la diabetes gestacional asociada a la condición de embarazo.

La diabetes, cualquiera que sea su tipo, afecta la salud de las personas y genera costos elevados para los sistemas de salud. En este estudio se hizo un seguimiento a sujetos con diabetes mellitus tipo 1, la cual se considera la condición más severa por ser insulino-dependiente. Una revisión de la fisiopatología de la diabetes mellitus tipo 1 puede ser consultada en la referencia [9] o en los libros de medicina interna. La acumulación de la glucosa en los órganos de la economía, en los intersticios conectivos y alrededor de los capilares genera procesos de inflamación y un incremento en la susceptibilidad a las infecciones; lo anterior puede afectar la condición de salud oral y periodontal $[10,11]$.

Los estudios previos indican una alta prevalencia deperiodontitis en pacientes con diabetes mellitus tipo 2 y una razón de riesgo (Odds ratio, oR) 2,6 veces mayor cuando se comparan con no diabéticos $[12,13]$. No obstante, la importancia del efecto de la diabetes en la enfermedad periodontal, independientemente de su tipo, es resaltada en estudios clínicos $[14,15]$.

La prevalencia de la enfermedad periodontal se encuentra en los rangos de 22 a $68 \%$ para gingivitis y de 25 a $98 \%$ para periodontitis en sujetos diabéticos [16-18], lo cual indica que la diabetes puede ser un im- 
portante modulador de la inflamación periodontal y causa importante de la pérdida dental.

El objetivo de este estudio fue determinar la condición periodontal en un grupo de sujetos con diabetes mellitus tipo 1 o DM1 del Hospital Universitario San Vicente de Paúl de Medellín, y hace parte de un análisis secundario de un estudio previamente publicado [19].

Los pacientes pertenecieron a la Asociación de Diabéticos de Antioquia, del Hospital San Vicente de Paúl, entre enero de 2010 y febrero de 2011. Participaron de forma voluntaria en el estudio, que tuvo un aval ético institucional y siguió las recomendaciones para investigaciones con sujetos humanos del Ministerio de Salud, en su Resolución 8430 de 1993.

La naturaleza del estudio fue de tipo descriptivo de corte transversal, la muestra fue de 35 pacientes por conveniencia. Los sujetos con diabetes melitus tipo 1 tuvieron controles periódicos de su condición de glicemia por medio de la hemoglobina glicosilada, y además tuvieron consejería para el manejo de la dieta y un control médico regular. Los criterios de inclusión y de exclusión fueron reportados en el artículo publicado [19].

\section{Evaluación clínica periodontal}

El examen periodonto fue practicado por periodoncistas calibrados para la valoración periodontal con una alta concordancia, con un valor kappa entre 0,80 y 0,90 .

Se registraron los siguientes parámetros periodontales en seis sitios en cada diente: profundidad a la son$\mathrm{da}(\mathrm{mm})$, pérdida de inserción clínica $(\mathrm{mm})$, porcentaje de sitios con sangrado a la sonda (\%) e índice de placa bacteriana (\%) [20], y número de dientes presentes y ausentes. El sondeo periodontal se hizo con una sonda periodontal UNC-15 (USA Delta, Chicago IL, USA).

Se determinó el diagnóstico periodontal según las recomendaciones de la American Academy of Periodontology [21, 22] y de Page y Eke [23].

El diagnóstico de gingivitis incluyó la presencia de, al menos, cuatro sitios en diferentes dientes que presentaban mínima profundidad al sondeo $(\leq 3 \mathrm{~mm})$, con o sin sangrado, pero sin presentar pérdida de inserción clínica y con ausencia radiográfica de pérdida ósea. Los sujetos con gingivitis podían presentar un periodonto reducido. La periodontitis crónica se diagnosticó por la presencia de, al menos, dos sitios proximales en diferentes dientes con bolsas ( $\geq 4 \mathrm{~mm}$ ), pérdida de inserción clínica ( $\geq 4 \mathrm{~mm}$ ), sangrado al sondeo y pérdida ósea observable radiográficamente. La pérdida ósea se evidenció en radiografías de aleta de mordida.
La duración de la diabetes, la historia familiar de diabetes, los medicamentos usados (insulina, hipoglucemiantes orales y otros), los hábitos de higiene oral y la frecuencia de complicaciones sistémicas relacionadas con la diabetes fueron también registrados [19].

\section{Análisis estadístico}

Para el análisis de datos, se realizó un descriptivo univariado para los indicadores clínicos. Además, se utilizó la prueba ANOva para comparar promedios en los cuatro diagnósticos periodontales, de dientes perdidos, sangrado, profundidad de bolsa, nivel de inserción, glicemia y HbA1C. Adicionalmente, se realizó una prueba $\mathrm{T}$ para comparar cada variable en dos grupos (periodontitis y gingivitis).

\section{Resultados}

Se incluyeron 35 sujetos con DM1 con una edad promedio de 50 años, de los cuales $62,9 \%$ fueron de sexo femenino (tabla 1); el promedio de edad de los sujetos fue 50,89 años con rango de edad entre los 19 y 75 años; el valor promedio de $\mathrm{HbAlC}$ fue de $8,574 \%$ entre un mínimo de 5,8 y un máximo de 16,4; la media del número de dientes perdidos fue 6,91 . El control de placa fue pobre $(55,67 \%)$ y debido a esta deficiente salud oral, se encontraron altos valores de sangrado $(55,22 \%)$; la pérdida de inserción promedio fue de $3,15 \mathrm{~mm}$, en la que el $25,7 \%$ presentó gingivitis; y de estas el más común fue la gingivitis localizada y un 74,3\% periodontitis crónica en sus formas localizada y generalizada (tablas 1 y 2 ).

El sangrado al sondeo (вор; 82,2\%), el promedio de profundidad de bolsa $(3,82 \mathrm{~mm})$ y el nivel de pérdida de inserción clínica $(4,2 \mathrm{~mm})$ fueron mayores en los pacientes con periodontitis generalizada, y estas diferencias fueron estadísticamente significantes al compararlas con los demás diagnósticos periodontales (tabla 2).

Tabla 1. Diagnóstico periodontal y de género en DM1

\begin{tabular}{|l|c|c|}
\hline & Frecuencia & Porcentaje \\
\hline Femenino & 22 & 62,9 \\
\hline Masculino & 13 & 37,1 \\
\hline Gingivitis localizada & 5 & 14,3 \\
\hline Gingivitis generalizada & 4 & 11,4 \\
\hline Periodontitis localizada & 18 & 51,4 \\
\hline Periodontitis generalizada & 8 & 22,9 \\
\hline
\end{tabular}

* Frecuencia: número de casos. Porcentaje: \% sobre un 100\%

Fuente: elaboración propia 
Tabla 2. Parámetros clínicos periodontales y de control de glicemia de la población con DM1

\begin{tabular}{|c|c|c|c|c|c|}
\hline & & $\mathrm{N}$ & $\mathrm{X}$ & $\pm \mathrm{SD}$ & $p \leq 0.05$ \\
\hline \multirow[t]{5}{*}{ n_dientes } & Gingivitis localizada & 5 & 19,60 & 7,436 & ,395 \\
\hline & Gingivitis generalizada & 4 & 25,25 & 4,272 & \\
\hline & periodontitis localizada & 18 & 20,72 & 4,586 & \\
\hline & Periodontitis generalizada & 8 & 20,75 & 5,471 & \\
\hline & Total & 35 & 21,09 & 5,221 & \\
\hline \multirow[t]{5}{*}{ dient_per } & Gingivitis localizada & 5 & 8,40 & 7,436 & ,395 \\
\hline & Gingivitis generalizada & 4 & 2,75 & 4,272 & \\
\hline & Periodontitis localizada & 18 & 7,28 & 4,586 & \\
\hline & Periodontitis generalizada & 8 & 7,25 & 5,471 & \\
\hline & Total & 35 & 6,91 & 5,221 & \\
\hline \multirow[t]{5}{*}{ BOP } & Gingivitis localizada & 5 & 22,360 & 5,5653 &, 000 \\
\hline & Gingivitis generalizada & 4 & 51,650 & 23,9573 & \\
\hline & Periodontitis localizada & 18 & 52,933 & 24,5492 & \\
\hline & Periodontitis generalizada & 8 & 82,675 & 12,6874 & \\
\hline & Total & 35 & 55,217 & 27,0033 & \\
\hline \multirow[t]{5}{*}{ PROMEDIO PD } & Gingivitis localizada & 5 & 1,9680 & ,23382 &, 000 \\
\hline & Gingivitis generalizada & 4 & 1,9925 &, 26387 & \\
\hline & Periodontitis localizada & 18 & 2,7978 &, 50871 & \\
\hline & Periodontitis generalizada & 8 & 3,8275 & 42108 & \\
\hline & Total & 35 & 2,8226 & ,77774 & \\
\hline \multirow[t]{5}{*}{ PD 1.3 NÚMERO } & Gingivitis localizada & 5 & 117,600 & 44,6184 &, 000 \\
\hline & Gingivitis generalizada & 4 & 149,750 & 24,3362 & \\
\hline & Periodontitis localizada & 18 & 103,389 & 25,2683 & \\
\hline & Periodontitis generalizada & 8 & 57,500 & 25,7071 & \\
\hline & Total & 35 & 100,229 & 38,8527 & \\
\hline \multirow[t]{5}{*}{ PD $1-3 \%$} & Gingivitis localizada & 5 & 100,000 &, 0000 &, 000 \\
\hline & Gingivitis generalizada & 4 & 98,950 & 2,1000 & \\
\hline & Periodontitis localizada & 18 & 84,294 & 15,2910 & \\
\hline & Periodontitis generalizada & 8 & 45,750 & 14,7124 & \\
\hline & Total & 35 & 79,403 & 23,4167 & \\
\hline \multirow[t]{5}{*}{ PD 4 - 5 NÚMERO } & Gingivitis localizada & 5 &, 000 &, 0000 &, 000 \\
\hline & Gingivitis generalizada & 4 & 1,750 & 3,5000 & \\
\hline & Periodontitis localizada & 18 & 16,611 & 18,1437 & \\
\hline & Periodontitis generalizada & 8 & 52,875 & 18,1772 & \\
\hline & Total & 35 & 20,829 & 24,3183 & \\
\hline \multirow[t]{5}{*}{ PD $4-5 \%$} & Gingivitis localizada & 5 &, 000 &, 0000 &, 000 \\
\hline & Gingivitis generalizada & 4 & 1,050 & 2,1000 & \\
\hline & Periodontitis localizada & 18 & 12,517 & 11,5219 & \\
\hline & Periodontitis generalizada & 8 & 43,013 & 10,8213 & \\
\hline & Total & 35 & 16,389 & 18,2421 & \\
\hline \multirow[t]{5}{*}{ NIVEL INSER } & Gingivitis localizada & 5 & 2,3740 & ,43183 & ,003 \\
\hline & Gingivitis generalizada & 4 & 2,1350 & ,43401 & \\
\hline & Periodontitis localizada & 18 & 3,1222 & 1,02261 & \\
\hline & Periodontitis generalizada & 8 & 4,2263 & 1,26328 & \\
\hline & Total & 35 & 3,1549 & 1,17161 & \\
\hline \multirow[t]{3}{*}{ CAL 1- 3 NÚMERO } & Gingivitis localizada & 5 & 107,400 & 55,8552 & 009 \\
\hline & Gingivitis generalizada & 4 & 148,250 & 24,1713 & \\
\hline & Periodontitis localizada & 18 & 90,111 & 40,4677 & \\
\hline
\end{tabular}


Cont.

\begin{tabular}{|c|c|c|c|c|c|}
\hline & Periodontitis generalizada & 8 & 57,375 & 39,9891 & \\
\hline & Total & 35 & 91,743 & 47,5149 & \\
\hline \multirow[t]{5}{*}{ CAL $1-3 \%$} & Gingivitis localizada & 5 & 86,140 & 17,1430 & ,001 \\
\hline & Gingivitis generalizada & 4 & 98,000 & 3,4264 & \\
\hline & Periodontitis localizada & 18 & 70,156 & 23,5857 & \\
\hline & Periodontitis generalizada & 8 & 42,750 & 23,1100 & \\
\hline & Total & 35 & 69,357 & 26,9919 & \\
\hline \multirow[t]{5}{*}{ CAL 4-5 NÚMERO } & Gingivitis localizada & 5 & 9,200 & 9,9850 & 000 \\
\hline & Gingivitis generalizada & 4 & 3,250 & 5,8523 & \\
\hline & Periodontitis localizada & 18 & 24,444 & 14,4557 & \\
\hline & Periodontitis generalizada & 8 & 41,250 & 16,9432 & \\
\hline & Total & 35 & 23,686 & 18,1995 & \\
\hline \multirow[t]{5}{*}{ CAL $4-5 \%$} & Gingivitis localizada & 5 & 12,420 & 15,1685 &, 002 \\
\hline & Gingivitis generalizada & 4 & 2,000 & 3,4264 & \\
\hline & Periodontitis localizada & 18 & 21,306 & 13,6303 & \\
\hline & Periodontitis generalizada & 8 & 32,588 & 10,2208 & \\
\hline & Total & 35 & 20,409 & 15,0693 & \\
\hline
\end{tabular}

${ }^{*}$ La tabla anterior presenta la aplicación de pruebas ANOVA

Fuente: elaboración propia

El promedio de sondeo en pacientes con gingivitis fue de $1,97 \mathrm{~mm}$ y de $3,11 \mathrm{~mm}$ con periodontitis; el nivel de inserción fue de $2,26 \mathrm{~mm}$ en gingivitis y de 3,4 en periodontitis (tabla 3). El valor promedio de hemoglobina $\mathrm{HbA1C}$ de los pacientes diagnosticados con gingivitis fue de 9,15 , mientras que en pacientes con periodontitis fue de 8,375 . Se encontró un nivel de glicemia en un rango de 117 a $454 \mathrm{mg} / \mathrm{dl}$ en los individuos estudiados. Lo anterior muestra un pobre control glicémico (tablas 3 y 4 ).
Se encontró diferencia significativa entre gingivitis y periodontitis $p=, 000$ en el nivel de inserción clínica, y $p=, 000$ en el promedio PD (tabla 3 ).

Para comparar el promedio de cada una de las variables involucradas (están en la primera columna), se aplica esta prueba debido a que se tienen cuatro grupos: gingivitis localizada, gingivitis generalizada, periodontitis localizada y periodontitis generalizada.

De acuerdo con el valor de $p$, que se encuentra en la última columna, todas las variables, excepto número de dientes y dientes perdidos, presentan diferencias en sus promedios en alguno de los grupos formados.

Tabla 3. Comparación de parámetros clínicos y de control de glicemia en DM 1 entre gingivitis y periodontitis

\begin{tabular}{|l|l|r|r|r|c|}
\hline & \multicolumn{1}{|c|}{ Dx simplificado } & N & X & \pm SD & Sig. (bilateral) \\
\hline \multirow{2}{*}{ PROMEDIO PD } & Gingivitis & 9 & 1,9789 &, 23154 &, 000 \\
\cline { 2 - 6 } & Periodontitis & 26 & 3,1146 &, 67862 &, 42396 \\
\hline \multirow{2}{*}{ NIVEL INSER } & Gingivitis & 9 & 2,2678 & 1,19498 &, 000 \\
\cline { 2 - 6 } & Periodontitis & 26 & 3,4619 & 102,9442 & 0,792 \\
\hline \multirow{2}{*}{ GLICEMIA mg/DL } & Gingivitis & 9 & 239,667 & 102,1854 & 0,467 \\
\cline { 2 - 6 } & Periodontitis & 9 & 229,115 & 3,0710 & \\
\hline \multirow{2}{*}{ HbA1C } & Gingivitis & 26 & 8,150 & 2,6027 & \\
\cline { 2 - 6 } & Periodontitis & & & & \\
\end{tabular}

${ }^{*} \mathrm{PD}$ : profundidad de bolsa. N: número de sujetos

Fuente: elaboración propia 
Tabla 4. Prueba de muestras independientes

\begin{tabular}{|c|c|c|c|c|c|c|}
\hline & & \multicolumn{2}{|c|}{$\begin{array}{l}\text { Prueba de Levene para la } \\
\text { igualdad de varianzas }\end{array}$} & \multicolumn{3}{|c|}{ Prueba T para la igualdad de medias } \\
\hline & & $\mathrm{F}$ & Sig. & $\mathrm{t}$ & gl & Sig. (bilateral) \\
\hline \multirow[t]{2}{*}{ PROMEDIO PD } & Se han asumido varianzas iguales & 11,086 & ,002 & $-4,882$ & 33 &, 000 \\
\hline & No se han asumido varianzas iguales & & & $-7,382$ & 32,985 &, 000 \\
\hline \multirow[t]{2}{*}{ NIVEL INSER } & Se han asumido varianzas iguales & 4,808 &, 035 & $-2,911$ & 33 & ,006 \\
\hline & No se han asumido varianzas iguales & & & $-4,364$ & 32,895 &, 000 \\
\hline \multirow[t]{2}{*}{ GLICEMIA mg/DL } & Se han asumido varianzas iguales &, 174 & ,679 & ,267 & 33 & ,792 \\
\hline & No se han asumido varianzas iguales & & & ,266 & 13,871 & ,795 \\
\hline \multirow[t]{2}{*}{ HBA1C } & Se han asumido varianzas iguales &, 581 & ,452 &, 736 & 33 & 467 \\
\hline & No se han asumido varianzas iguales & & & ,678 & 12,230 &, 511 \\
\hline
\end{tabular}

${ }^{*}$ La tabla anterior corresponde a pruebas $\mathrm{T}$ para muestras independientes

Fuente: elaboración propia

Se están comparando los promedios de profundidad de bolsa, nivel de inserción clínica, glicemia y $\mathrm{HbA1C}$ en dos grupos: pacientes con gingivitis y pacientes con periodontitis.

Anteriormente, se muestra una tabla con resumen de descriptivos de cada una de las variables analizadas en los dos grupos, gingivitis y periodontitis (tabla 3). Según la tabla, en las dos primeras variables, la prueba T no asume varianzas iguales ( $\mathrm{P}$ de varianzas es 0,002 y 0,035 respectivamente). El valor de $p=0,000$ para promedio de profundidad de bolsa concluye que hay diferencia en los promedios de profundidad entre pacientes con gingivitis y pacientes con periodontitis.

Igual conclusión para el caso de la variable nivel de inserción.

Sin embargo, para glicemia y $\mathrm{HbA} 1 \mathrm{C}$ no se puede concluir que haya diferencia en los promedios considerando gingivitis y periodontitis. Los valores $p$ son 0,792 y 0,467 , respectivamente.

\section{Discusión}

En 35 sujetos con diabetes tipo 1 y sin tratamiento periodontal previo, se determinó la frecuencia de enfermedad periodontal $[19,24]$. La periodontitis fue muy prevalente $(74,3 \%)$, seguida por gingivitis $(25,7 \%), y$ ningún sujeto tuvo salud periodontal.

Se pudo comprobar que la enfermedad periodontal es más prevalente y más severa en los pacientes diabéticos $[2,3,10,17,19,25,26]$.

Como era de esperarse, los parámetros periodontales como sangrado al sondeo, profundidad de bolsa y pérdida de inserción clínica fueron más graves en los sujetos con periodontitis.

No hubo diferencia significativa entre gingivitis y periodontitis en el control de la glicemia; sin embargo, podemos afirmar que estos pacientes tuvieron un pobre control glicémico, como lo revelaron los valores promedio de glicemia en ayunas y de hemoglobina glicosilada (tablas 3 y 4 ).

Es importante anotar que los pacientes con gingivitis también pueden presentar un periodonto reducido pero sin bolsas periodontales. No es posible establecer, por la naturaleza del estudio de corte transversal, si estos sujetos ahora diagnosticados con gingivitis habían sufrido de periodontitis en el pasado [24].

Se reconoce que la diabetes mellitus tipo 1 es un factor predisponente para el desarrollo de la enfermedad periodontal [24].

En estos pacientes es importante instaurar una terapia periodontal y mejorar el control de la higiene bucal. La reparación periodontal en pacientes diabéticos sea con DM1 o DM2, particularmente en condiciones de pobre control metabólico de la enfermedad, manifiesta importantes deficiencias [21,22]. En consistencia con estas asociaciones, la hiperglicemia puede generar defectos en la neoformación ósea y la integración de implantes, debido a que se observa en modelos animales una respuesta hasta un 30\% menor en la formación de tejido óseo alrededor de implantes al ser comparados con controles [25, 27]. Por otro lado, al analizar los resultados de la instalación de implantes en humanos, los resultados muestran variaciones de entre un 0 y $15 \%$ de menor formación ósea en presencia de diabetes [2, 24]. Esta mayor divergencia de los resultados podría derivar de la amplia variabilidad en la evolución y control clínico de la diabetes 
en humanos. La conclusión general de estos estudios es que defectos en el control de la glicemia podrían explicar esta respuesta deficitaria en la reparación periodontal y periimplantaria en pacientes diabéticos [28].

La diabetes es una enfermedad que compromete la respuesta inflamatoria y reparativa del organismo. Es posible considerar además que los tejidos periodontales, al estar expuestos a un constante desafío infeccioso y traumático, podrían ser particularmente sensibles al efecto de la diabetes. La hiperglicemia podría actuar como mecanismo efector común de las alteraciones generadas por la diabetes en los tejidos periodontales. En este sentido, se ha observado que niveles elevados de glicemia pueden inhibir la proliferación celular en osteoblastos y la producción de colágeno durante las etapas tempranas de la formación de la reparación ósea, lo que resulta en una menor neoformación ósea y una reducida capacidad mecánica del hueso regenerado $[28,29]$.

Por otro lado, la modulación de la respuesta inflamatoria en diabéticos podría ser importante en la generación de las complicaciones descritas a nivel periodontal. Utilizando monocitos derivados de diabéticos tipo 1, Salvi et al. observaron que estas células respondían a lipopolisacáridos (LPS) de Porphyromonas gingivalis, liberando niveles aumentados de TNF- $\alpha$ en comparación con pacientes no diabéticos pareados por su estado periodontal [30]. Los diabéticos tipo 1 mostraron niveles incrementados de PGE2 e IL1 $\beta$ en el fluido crevicular, en comparación con controles no diabéticos pareados por severidad de enfermedad periodontal. Más aún, monocitos de estos pacientes estimulados con LPS de Escherichia coli y $P$. gingivalis liberaron niveles significativamente superiores de PGE2 e IL-1 $\beta$ en comparación con monocitos de pacientes no diabéticos con enfermedad periodontal. Estos estudios sugieren que los pacientes diabéticos pueden tener una respuesta inflamatoria más agresiva que pacientes no diabéticos a niveles comparables de enfermedad periodontal. En relación con la reparación periodontal, TGF- $\beta 1$ es un factor de crecimiento que promueve la producción de colágeno tipo 1 y la diferenciación de miofibroblastos, lo que facilita la regeneración de la matriz extracelular [30]. Es posible entonces que la respuesta inflamatoria en pacientes diabéticos pueda retardar la reparación y regeneración de tejidos a nivel periodontal.

Los pacientes con DM1 requieren prevención y tratamiento periodontal. Los primeros estudios en mostrar un efecto del tratamiento de la periodontitis en la diabetes fueron desarrollados por Grossi et al., y mostraron que el tratamiento de la periodontitis era capaz de reducir los niveles de HbA1C en la población de indios Pima [31]. Una serie de estudios han precedido este trabajo pionero y un meta-análisis que reunió la información derivada de 10 estudios de intervención mostró una reducción de $0,38 \%$ en los niveles de $\mathrm{HbA1C}$, de $0,66 \%$ al restringir el análisis a pacientes diabéticos tipo 2 y de $0,71 \%$, al considerar el uso de antibióticos durante el tratamiento. Sin embargo, estos valores de reducción en los niveles de hemoglobina glicosilada no alcanzaron niveles de significancia estadística [31]. Es posible que estas diferencias en el control metabólico de la diabetes, en respuesta al tratamiento de la enfermedad periodontal, se deban a la heterogeneidad de las poblaciones estudiadas [32-34]. La evidencia a la fecha permite pensar que el tratamiento de la enfermedad periodontal es capaz de contribuir al control de la glicemia. Sin embargo, la magnitud y significancia clínica de este efecto aún requiere de nuevos y mejores estudios.

\section{Conclusiones}

En conclusión, 26 de los 35 pacientes con DM1 presentaron periodontitis de adulto localizada. Ninguno de los pacientes presentó periodonto sano. Adicionalmente, estos pacientes presentaron un pobre control de la glicemia.

\section{Agradecimientos}

Este estudio fue financiado parcialmente por el proyecto de convocatoria interna CI 1695 de la Universidad del Valle 2012 "Genotipos de fima de Porphyromonas gingivalis asociados con la severidad de periodontitis en diabéticos". Los autores agradecen al profesor Javier Enrique Botero, docente de la Facultad de Odontología de la Universidad de Antioquia, por facilitar la información de los pacientes con DM1.

\section{Referencias}

[1] American Diabetes Association. Diagnosis and classification of diabetes mellitus. Diabetes Care. 2006;29(1): s43-s48.

[2] Lalla E, Kunzel C, Burkett S, Cheng B, Lamster IB. Identification of unrecognized diabetes and pre-diabetes in a dental setting. J Dent Res. 2011;90(7):855-60.

[3] Li S, Williams PL, Douglass CW. Development of a clinical guideline to predict undiagnosed diabetes in dental patients. J Am Dent Assoc. 2011;142(1):28-37. 
[4] Aschner P. Epidemiología de la diabetes en Colombia. Av Diabetol. 2010;26(2):95-100.

[5] Gurav A, Jadhav V. Periodontitis and risk of diabetes mellitus. J Diabetes. 2011;3(1):21-8.

[6] Moles DR. Evidence of an association between diabetes and severity of periodontal disease. Evid Based Dent. 2006; doi:10.1038/sj.ebd.6400409.

[7] Tervonen T, Oliver RC. Long-term control of diabetes mellitus and periodontitis. J Clin Periodontol. 1993;20(6):431-5.

[8] Westfelt E, Rylander H, Blohmé G, Jonasson P, Lindhe J. The effect of periodontal therapy in diabetics. Results after 5 years. J Clin Periodontol. 1996;23(2):92-100.

[9] Fauci A, Kasper DL, Braunwald E. Harrison: principios de medicina interna. Vol. 2. 16 a ed. México: McGrawHill / Interamericana; 2005.

[10] Mealey BL, Rose LF. Diabetes mellitus and inflammatory periodontal diseases. Curr Opin Endocrinol Diabetes Obes. 2008;15(2):135-41.

[11] Roy S, Trudeau K, Behl Y, Dhar S, Chronopoulos A. New insights into hyperglycemia-induced molecular changes in microvascular cells. J Dent Res. 2010;89(2):116-27.

[12] Emrich LJ, Shlossman M, Genco RJ. Periodontal disease in non-insulin-dependent diabetes mellitus. J Periodontol. 1991;62(2):123-31.

[13] Nelson RG, Shlossman M, Budding LM, Pettitt DJ, Saad MF, Genco RJ, et al. Periodontal disease and NIDDM in Pima Indians. Diabetes Care. 1990;13(8):836-40.

[14] Lalla E, Kaplan S, Chang SM, Roth GA, Celenti R, Hinckley K, et al. Periodontal infection profiles in type 1 diabetes. J Clin Periodontal. 2006;33(12):855-62.

[15] Ryan ME, Carnu O, Kamer A. The influence of diabetes on the periodontal tissues. J Am Dent Assoc. 2003;134: 34 S-40S.

[16] Linares-Vieyra C, González-Guevara MB, Rodríguezde Mendoza LE. Periodontal diseases and treatment needs in diabetes type 2. Rev Med Inst Mex Seguro Soc. 2009;47(2):129-34.

[17] Pavez V, Araya V, Rubio A, Ríos L, Meza P, Martínez B. Periodontal health status in subjects with diabetes mellitus type 1 , from 18 to 30 years-old, from Santiago de Chile. Rev Med Chil. 2002;130(4):402-8.

[18] World Health Organization. What is the burden of oral disease? [internet]. 2013 [citado 2011 abr 3]. Disponible en: http://www.who.int/oral_health/disease_burden/ global/en/

[19] Ochoa SP, Ospina CA, Colorado KJ, Montoya YP, Saldarriaga $\mathrm{AF}$, Galvis $\mathrm{M}$, et al. Condición periodontal y pérdida dental en pacientes diabéticos del Hospital Universitario San Vicente de Paúl. Biomédica. 2012;32(1):52-9.
[20] O'Leary TJ, Drake RB, Naylor JE. The plaque control record. J Periodontol. 1972;43(1):38.

[21] Mariott A. Dental plaque-induced gingival diseases. Ann Periodontol. 1999;4(1):7-9.

[22] Lindhe J, Ranney R, Lamster I, Charles A, Chung CP , Flemmig T, et al. Consensus report: chronic periodontitis. Ann Periodontol. 1999;4(1):38.

[23] Page RC, Eke PI. Case definitions for use in population-based surveillance of periodontitis. J Periodontol. 2007;78(7):1387-99.

[24] Botero JE, Yepes FL, Roldán N, Castrillón CA, Hincapie JP, Ochoa SP, et al. Tooth and periodontal clinical attachment loss are associated with hyperglycemia in patients with diabetes. J Periodontol. 2012;83(10):1245-50.

[25] Bascones-Martínez A, Arias-Herrera S, Criado-Cámara E, Bascones-Ilundáin J, Bascones-Ilundáin C. Periodontal disease and diabetes. Adv Exp Med Biol. 2012;771:76-87.

[26] Loe H. Periodontal disease. The sixth complication of diabetes mellitus. Diabetes Care. 1993;16(1):329-34.

[27] Moore PA, Weyant RJ, Mongelluzzo MB, Myers DE, Rossie K, Guggenheimer J, et al. Type 1 diabetes mellitus and oral health: Assessment of tooth loss and edentulism. J Public Health Dent. 1998;58(2):135-42.

[28] Beikler T, Flemmig TF. Implants in the medically compromised patient. Crit Rev Oral Biol Med. 2003;14(4):305-16.

[29] Fiorellini JP, Chen PK, Nevins M, Nevins ML. A retrospective study of dental implants in diabetic patients. Int J Periodontics Restorative Dent. 2000;20(4):366-73.

[30] Salvi GE, Yalda B, Collins JG, Jones BH, Smith FW, Arnold $\mathrm{RR}$, et al. Inflammatory mediator response as a potential risk marker for periodontal diseases in insulin-dependent diabetes mellitus patients. J Periodontol. 1997;68(2):127-35.

[31] Grossi SG, Skrepcinski FB, De Caro T, Robertson DC, Ho AW, Dunford RG, et al. Treatment of periodontal disease in diabetics reduces glycated hemoglobin. J Periodontol. 1997;68(8):713-9.

[32] Janket SJ, Wightman A, Baird AE, Van Dyke TE, Jones JA. Does periodontal treatment improve glycemic control in diabetics patients? A meta-analysis of interventional studies. J Dent Res. 2005;84(12):1154-9.

[33] Simpson TC, Needleman I, Wild SH, Moles DR, Mills EJ. Treatment of periodontal disease for glycaemic control in people with diabetes. Cochrane Database Syst Rev. 2010;12(5):CD004714.

[34] Vergnes JN. Treating periodontal disease may improve metabolic control in diabetics. Evid Based Dent. 2010;11(3):73-4. 